\title{
Tuned effector functions and adduct structures of an engineered human Fc fragment
}

\author{
Travis Gallagher ${ }^{\mathrm{a}}$, Chris McCullough ${ }^{\mathrm{b}}$, Robert Brinson ${ }^{\mathrm{c}}$, Joomi Ahn ${ }^{\mathrm{d}}$ and Nazzareno Dimasi ${ }^{\mathrm{e}}$ \\ a National Inst of Standards and Technology; 9600 Gudelsky Dr, Rockville, Maryland 20854, USA; \\ travis.gallagher@nist.gov \\ b NIST; mcculloughc@ibbr.umd.edu \\ ${ }^{c}$ NIST; brinson@ibbr.umd.edu \\ d1 Medimmune Way, Gaithersburg, Maryland 20878, USA; AhnJ@Medlmmune.com \\ e 1 Medimmune Way, Gaithersburg, Maryland 20878, USA; DimasiN@MedImmune.com
}

Controlled conjugation of chemical moieties onto monoclonal antibodies (mAbs) can enable the delivery of therapeutic functionalities to specific tissues. The conjugation method and site on the protein have important consequences for the stability of the construct, and for its capacity to bind to cellular receptors that mediate its biological trajectory and thus its therapeutic effects. We report the crystal structure and dynamics as measured by HDX-MS (hydrogen-deuterium exchange mass spectrometry) of a human Fc fragment with a single, site-specifically inserted cysteine ( $\mathrm{Fc}-\mathrm{C} 239 \mathrm{i})$ with two different adducts attached to the inserted cysteine as proto linkers. The $2.3 \AA$ and $2.7 \AA$ resolution data (for the cysteine and maleimide-PEG adducts, respectively) show that the inserted cysteine structurally replaces serine 239, projecting the linker into a cleft adjacent to the glycan. The insertion causes an upward shift in the local beta strand, effectively pushing Ser 239 into the location normally occupied by Pro 238, pushing Pro 238 into the location of Gly 237, and extending the length of the hinge by one residue as compared to wild-type. The linker site is highly constrained and shielded in the cleft, adjacent to the glycan. This protected location is consistent with its reported stability. The structures, in comparison with wild-type FcyRIIla complexes, show how the insertion at this site alters the hinge conformation in a way that would prevent FCyRIIla binding, thus explaining the previously observed elimination of ADCC activity for $\mathrm{Fc}-\mathrm{C} 239 \mathrm{i}$. However, despite a general increase in $\mathrm{CH} 2$-domain dynamics, the binding site for FcRn appears unaffected, consistent with FcRn binding measurements. Both results (abolition of FcyRIIla binding and retention of FcRn binding) are favorable to the design of antibody-drug conjugate medicines. 